\title{
RT-qPCR analysis of the tumor antigens TOMM34 and RNF43 in samples extracted from paraffin-embedded specimens of colorectal cancer
}

\author{
NORIMASA MATSUSHITA $^{1}$, SHIHO YAMAMOTO ${ }^{1}$, YUJI INOUE $^{1}$, \\ ATSUSHI ARUGA $^{2}$ and MASAKAZU YAMAMOTO ${ }^{1}$ \\ ${ }^{1}$ Department of Gastroenterological Surgery, ${ }^{2}$ Institute of Advanced Biomedical Engineering and Science, \\ Tokyo Women's Medical University, Tokyo 162-8666, Japan \\ Received November 1, 2016; Accepted April 28, 2017
}

DOI: $10.3892 / \mathrm{ol} .2017 .6412$

\begin{abstract}
Comprehensive genetic analysis of colorectal malignant tumors by microarrays has identified translocase of the outer mitochondrial membrane 34 (TOMM34) and ring finger protein 43 (RNF43) as highly expressed oncogenes in malignant colorectal tumors. Vaccine therapy using cancer peptides synthesized using the amino acid sequences of tumor antigens is currently undergoing clinical trials. Since it is important to perform vaccine therapy based on the oncogene expression levels in individual tumors, analysis of tumor antigen expression is necessary for this therapy. However, the quality of the messenger RNA extracted from formalin-fixed and paraffin-embedded specimens is generally considered insufficient for gene quantification. The present study examined whether it could be possible to quantify the expression of TOMM34 and RNF43 in colorectal cancer and liver metastasis samples prepared from paraffin blocks. The formalin-fixed and paraffin-embedded specimens were sliced for slides and the colorectal cancer and normal mucosal tissues were obtained from the slides. Total RNA was extracted from the tissue samples, and quantitative polymerase chain reaction (qPCR) was performed using the Universal ProbeLibrary as a PCR probe. Quantification of TOMM34 and RNF43 gene expression in several-year-old paraffin-embedded colorectal cancer specimens was possible by qPCR using the Universal ProbeLibrary. qPCR revealed that TOMM34 expression was elevated in $78.9 \%$ (15 of 19 cases) of the primary tumors and in $73.7 \%$ (14 of 19 cases) of the liver metastasis samples. RNF43 expression was elevated in $63.2 \%$ (12 of 19 cases) of
\end{abstract}

Correspondence to: Dr Norimasa Matsushita, Department of Gastroenterological Surgery, Tokyo Women's Medical University, 8-1 Kawada-cho, Shinjuku-ku, Tokyo 162-8666, Japan

E-mail: matsushita0710@hotmail.com

Key words: RT-qPCR, TOMM34, RNF43, colorectal cancer, Universal ProbeLibrary the primary tumors and in $73.7 \%$ (14 of 19 cases) of the liver metastasis samples.

\section{Introduction}

Previous comprehensive genetic analyses of colorectal cancer by microarrays identified translocase of the outer mitochondrial membrane 34 (TOMM34) and ring finger protein 43 (RNF43) as oncogenes expressed in colorectal cancer $(1,2)$. TOMM34 and RNF43 are considered to be associated with cell proliferation, and clinical studies of vaccine therapy with an artificially synthesized cancer peptide based on the amino acid sequence of tumor antigens such as TOMM34 and RNF43 are ongoing (3-6). A tumor antigen inoculated by vaccine is used for antigen presentation from dendritic cells to lymphocytes, and cytotoxic T-lymphocytes (CTLs) are induced in vivo. The induced CTLs are expected to recognize the tumor antigen and show an antitumor effect against cancer cells expressing the tumor antigen. Since it is important that vaccine therapy should be performed based on the expression levels of tumor antigens in an individual for the effective treatment, analysis of oncogene expression is essential. Formalin-fixed, paraffin-embedded (FFPE) tissue samples are the most available material for pathological studies. However, the quality of mRNA extracted from FFPE specimens is generally considered to be insufficient for gene analyses due to nucleic acid degradation (7). The present study examined methodologies for the quantification of TOMM34 and RNF43 in tissue samples extracted from FFPE colorectal cancer specimens. The samples used in the present study were specimens from 19 patients with colorectal cancer and liver metastasis, and the tumors were surgically removed in the Department of Gastroenterological Surgery of Tokyo Women's Medical University (Tokyo, Japan) between December 2004 and October 2008. The FFPE samples were prepared from the resected tumors and stored at room temperature until present examination. The tissue slides were prepared from the FFPE samples and total RNA was extracted from the slides. Subsequent to synthesizing cDNA from the extracted total RNA, quantitative polymerase chain reaction was performed using the Universal ProbeLibrary as a PCR probe to measure 
the gene expression levels of TOMM34 and RNF43 in the several-year-old colorectal FFPE samples.

\section{Materials and methods}

Subjects. Samples were obtained from 19 cases of colorectal cancer with liver metastasis that were removed surgically at the Department of Gastroenterological Surgery of Tokyo Women's Medical University between December 2004 and October 2008. The tissue samples were paraffin embedded upon formalin fixation and stored at room temperature until examination. As a negative control for the expression of TOMM34 and RNF43, peripheral blood mononuclear cells (PBMCs) were obtained from healthy 35-year-old male. The present study was approved by the Institutional Review Board of Tokyo Women's Medical University. Informed consent was obtained from the subjects for the use of their tissues.

Patient characteristics. As shown in Table I, the patients' ages ranged from 52 to 78 years (average, 65.1 years). There were 14 males and 5 females. The histological type of the primary tumors was well differentiated adenocarcinoma in 15 patients and moderately differentiated adenocarcinoma in 4 patients. The site of the primary tumor was the sigmoid colon in $8(42 \%)$ cases, the ascending colon in $6(32 \%)$ cases, the cecum in $3(16 \%)$ cases, the rectum in $1(5 \%)$ case and the transverse colon in $1(5 \%)$ case.

Colon cancer CW2 cell line. The human colon cancer cell line CW2 (RIKEN BioResource Center, Tsukuba, Japan) was selected as a positive control for the expression of TOMM34 and RNF43. CW2 cells were obtained from a 60-year-old female who underwent the surgical removal of colon cancer, and whose case was described (8). The cell line was maintained in medium consisting of RPMI-1640 (Thermo Fisher Scientific, Inc., Waltham, MA, USA) containing 10\% heat-inactivated fetal bovine serum (Thermo Fisher Scientific, Inc.), $100 \mathrm{U} / \mathrm{ml}$ penicillin and $100 \mu \mathrm{g} / \mathrm{ml}$ streptomycin. The cell line was cultured at $37^{\circ} \mathrm{C}$ in a $95 \%$ humidity atmosphere containing $5 \% \mathrm{CO}_{2}$.

Tissue sampling and extraction of RNA. For the extraction of total RNA from CW2 and PBMCs of a healthy 35-year-old male donor (mentioned in Subjects paragraph), the RNeasy Mini kit (Qiagen, Inc., Valencia, CA, USA) was used. From surgically resected specimens, separate slides of normal and cancer tissue were prepared. The normal mucosa was obtained from surgical edge of the resected colon which site was at least $10 \mathrm{~cm}$ away from the tumor. Hematoxylin and eosin (H\&E)-stained slides were examined, and the normal and cancer tissue areas in the slides were confirmed prior to all experimental procedures. No cancer tissue contaminated the normal tissue slides, which was confirmed by pathologists. For the extraction of total RNA from paraffin-embedded specimens, cancer and normal mucosa (mucosa without tumor) specimens were obtained from slides of the paraffin blocks previously examined under H\&E staining, and total RNA was extracted upon deparaffinization using an RNeasy FFPE kit (Qiagen, Inc.). All steps were performed according to the manufacturer's protocol.
Reverse transcription-polymerase chain reaction (RT-PCR). Qualitative RT-PCR using 50 ng total RNA from CW2 or PBMCs of a 35-year-old male healthy donor (mentioned in Subjects paragraph) was performed with Ready-To-Go RT-PCR Beads (GE Healthcare Life Sciences, Little Chalfont, UK) following the manufacturer's protocol. The 5'-3' sequences of the primers used for the reaction were as follows: TOMM34-forward (F), TTG CAG ACA TCA GCA ACC TC; TOMM34-reverse (R), ACC TTT CTG GTG CAA CAA CC; RNF43-F, AAA GGA CCA CAG CAA ACA CC; RNF43-R, CTG AAC CCA CTG GCT GGT AT; GAPDH-F, CGA CCA CTT TGT CAA GCT CA; and GAPDH-R, TGT GAG GAG GGG AGA TTC AG. The conditions of the PCR were as follows: $95^{\circ} \mathrm{C}$ for $5 \mathrm{~min}, 35$ cycles of $95^{\circ} \mathrm{C}$ for $30 \mathrm{sec}$, $56^{\circ} \mathrm{C}$ for $30 \mathrm{sec}$ and $72^{\circ} \mathrm{C}$ for $30 \mathrm{sec}$, and $72^{\circ} \mathrm{C}$ for $5 \mathrm{~min}$. The PCR products were evaluated by $1 \%$ agarose (Sigma-Aldrich; Merck KGaA, Darmstadt, Germany) gel electrophoresis and ethidium bromide (Sigma-Aldrich; Merck KGaA) staining.

Probes and primers for quantitative PCR (qPCR) and PCR conditions. The Universal ProbeLibrary (Roche Diagnostics, Basel, Switzerland) was selected for qPCR analysis, and various primers and probes were selected using ProbeFinder software version 2.43 from the human set of the Universal ProbeLibrary Assay Design Center (https://qpcr.probefinder. com/organism.jsp). The sequences $\left(5^{\prime}-3^{\prime}\right)$ of the primers for TOMM34 were as follows: TOMM34-F, CAA ATC CAA AGA AAC CAC AGC; and TOMM34-R, AGA ACT CTG GCT TTC TCC ACA, and the probe number was 3. The sequences (5'-3') of the primers for RNF43 were as follows: RNF43-F, TTA TCC GCA CTG CCA GGT; and RNF43-R, CAC AGC TCC TCG AGT TCC TC, and the probe number was 64 . The sequences $\left(5^{\prime}-3^{\prime}\right)$ of the primers for GAPDH were as follows: GAPDH-F, AGC CAC ATC GCT CAG ACA C; and GAPDH-R, GCC CAA TAC GAC CAA ATC C, and the probe number was 60. Complementary DNA (cDNA) was obtained using SuperScript III First-Strand Synthesis SuperMix for qRT-PCR (Invitrogen; Thermo Fisher Scientific, Inc., Waltham, MA, USA) with total RNA (50 ng) extracted from the paraffin-embedded specimens. qPCR was performed with this cDNA using LightCycler Taqman Master in a LightCycler Instrument (both from Roche Diagnostics). All reactions were conducted following the manufacturer's protocol. The PCR conditions were as follows: $95^{\circ} \mathrm{C}$ for $10 \mathrm{~min}$, followed by 45 cycles of $95^{\circ} \mathrm{C}$ for $10 \mathrm{sec}, 60^{\circ} \mathrm{C}$ for $10 \mathrm{sec}$ and $72^{\circ} \mathrm{C}$ for $10 \mathrm{sec}$.

Genetic sequences, relative quantification of gene expression and statistical analysis. The genetic sequences for TOMM34, RNA43 and GAPDH were deposited in GenBank (https://www. ncbi.nlm.nih.gov/genbank/). The accession numbers were as follows: TOMM34 (NM_006809.4), RNF43 (NM_017763.4) and GAPDH (NM_002046.3).

For the relative quantification of target gene expression, a previously reported calculation method was used (9). The point at which the fluorescence of a sample exceeded that of the background was identified as a crossing point (CP) in the amplification process of the PCR. The CP value of the reference gene GAPDH was subtracted from the $\mathrm{CP}$ value of each sample, and this value was defined as $\triangle \mathrm{CP}$. The $\triangle \mathrm{CP}$ of the 
Table I. Characteristics of 19 patients with colorectal cancer and histological type of primary tumors.

\begin{tabular}{|c|c|c|c|c|}
\hline Patient no. & Age, years & Sex & Tumor site & Histological type of primary tumor \\
\hline 1 & 65 & Male & $\mathrm{R}$ & Moderately differentiated adenocarcinoma \\
\hline 2 & 70 & Male & $\mathrm{S}$ & Well differentiated adenocarcinoma \\
\hline 3 & 64 & Male & A & Well differentiated adenocarcinoma \\
\hline 4 & 64 & Male & $\mathrm{S}$ & Well differentiated adenocarcinoma \\
\hline 5 & 54 & Male & A & Well differentiated adenocarcinoma \\
\hline 6 & 69 & Male & $\mathrm{S}$ & Well differentiated adenocarcinoma \\
\hline 7 & 78 & Male & $\mathrm{S}$ & Well differentiated adenocarcinoma \\
\hline 8 & 59 & Female & A & Well differentiated adenocarcinoma \\
\hline 9 & 69 & Male & $\mathrm{S}$ & Well differentiated adenocarcinoma \\
\hline 10 & 75 & Male & $\mathrm{C}$ & Well differentiated adenocarcinoma \\
\hline 11 & 72 & Female & A & Moderately differentiated adenocarcinoma \\
\hline 12 & 56 & Female & $\mathrm{C}$ & Moderately differentiated adenocarcinoma \\
\hline 13 & 52 & Female & $\mathrm{S}$ & Well differentiated adenocarcinoma \\
\hline 14 & 55 & Male & A & Moderately differentiated adenocarcinoma \\
\hline 15 & 64 & Male & $\mathrm{C}$ & Well differentiated adenocarcinoma \\
\hline 16 & 70 & Female & A & Well differentiated adenocarcinoma \\
\hline 17 & 62 & Male & $\mathrm{T}$ & Well differentiated adenocarcinoma \\
\hline 18 & 72 & Male & $\mathrm{S}$ & Well differentiated adenocarcinoma \\
\hline 19 & 68 & Male & $\mathrm{S}$ & Well differentiated adenocarcinoma \\
\hline
\end{tabular}

C, cecum; A, ascending colon; T, transverse colon; S, sigmoid colon; R, rectum.

colon cancer cell line CW2 used as a control was subtracted from the $\triangle \mathrm{CP}$ of each sample, and the resulting value was defined as $\Delta \Delta \mathrm{CP}$. The relative gene expression level of each sample was calculated as $2^{-\Delta \Delta C P}$, and the data obtained were used for further evaluation of gene expression. The gene expression data was collected using Microsoft Excel 2013 (Microsoft, Redmond, WA, USA) and was statistical analyzed using IBM SPSS Statistics 21 (IBM SPSS, Armonk, NY, USA). The Student's t-test was performed to compare the results between two groups. $\mathrm{P}<0.05$ was considered to indicate a statistically significant difference.

\section{Results}

Qualitative PCR of the colon cancer cell line CW2. Total RNA was extracted from CW2 cells and used in qualitative PCR (Fig. 1). TOMM34 and RNF43 expression was detected in CW2 cells but no in PBMCs. Based on these results, CW2 cells were selected as a positive control.

Relative gene expression level of each sample. The $2^{-\Delta \Delta \mathrm{CP}}$ data of the normal mucosa, primary tumor and liver metastasis are summarized in Table II. TOMM34 expression was elevated, compared with that in the normal mucosa, in 15 of the 19 primary tumors $(78.9 \%)$ and in 14 of the 19 samples of liver metastasis (73.7\%). The graph depicted in Fig. 2A indicates mean and SD of TOMM34 expression in normal mucosa, primary tumor and liver metastasis obtained from all patients showed higher expression in the primary tumors and liver metastases compared with the gene expression in the normal mucosa, but the difference was not significant. Similarly,

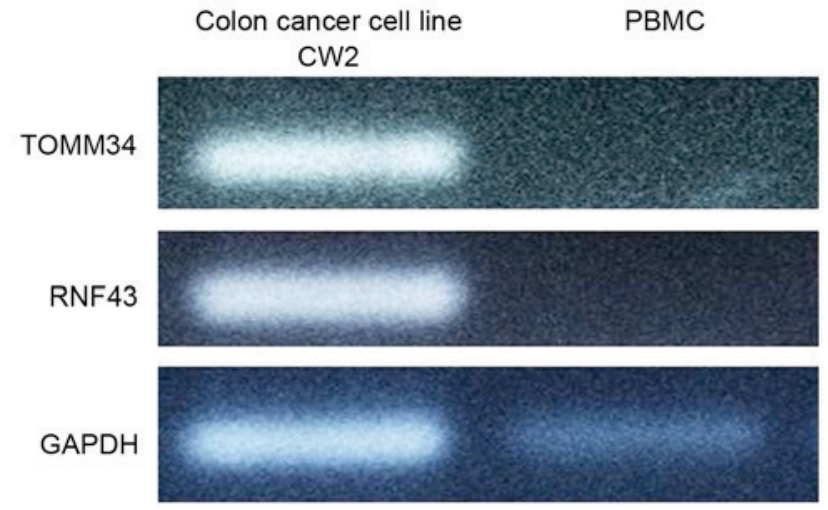

Figure 1. Qualitative evaluation of TOMM34 and RNF43 expression in the colorectal cancer cell line CW2 and PBMCs of healthy donor by reverse transcription-polymerase chain reaction. PBMC, peripheral blood mononuclear cell; TOMM34, translocase of the outer mitochondrial membrane 34; RNF43, ring finger protein 43.

RNF43 exhibited higher expression, compared with that in the normal mucosa, in 12 of the 19 primary tumors $(63.2 \%)$ and in 14 of the 19 liver metastases $(73.7 \%)$. The $2^{-\Delta \Delta C P}$ values of RNF43 in the primary tumors and liver metastases were compared with those in the normal mucosa. The expression of RNF43 in the liver metastases was significantly higher $(\mathrm{P}<0.05)$ than that in the normal mucosa (Fig. 2A). TOMM34 and RNF43 did not show significant differences in relative gene expression level (Fig. 2A) between their expression in primary tumors and liver metastases. The gene expression level of all patients' primary tumors and liver metastases were plotted in Fig. 2B. Fig. 2C showed enlarged figure of squared 


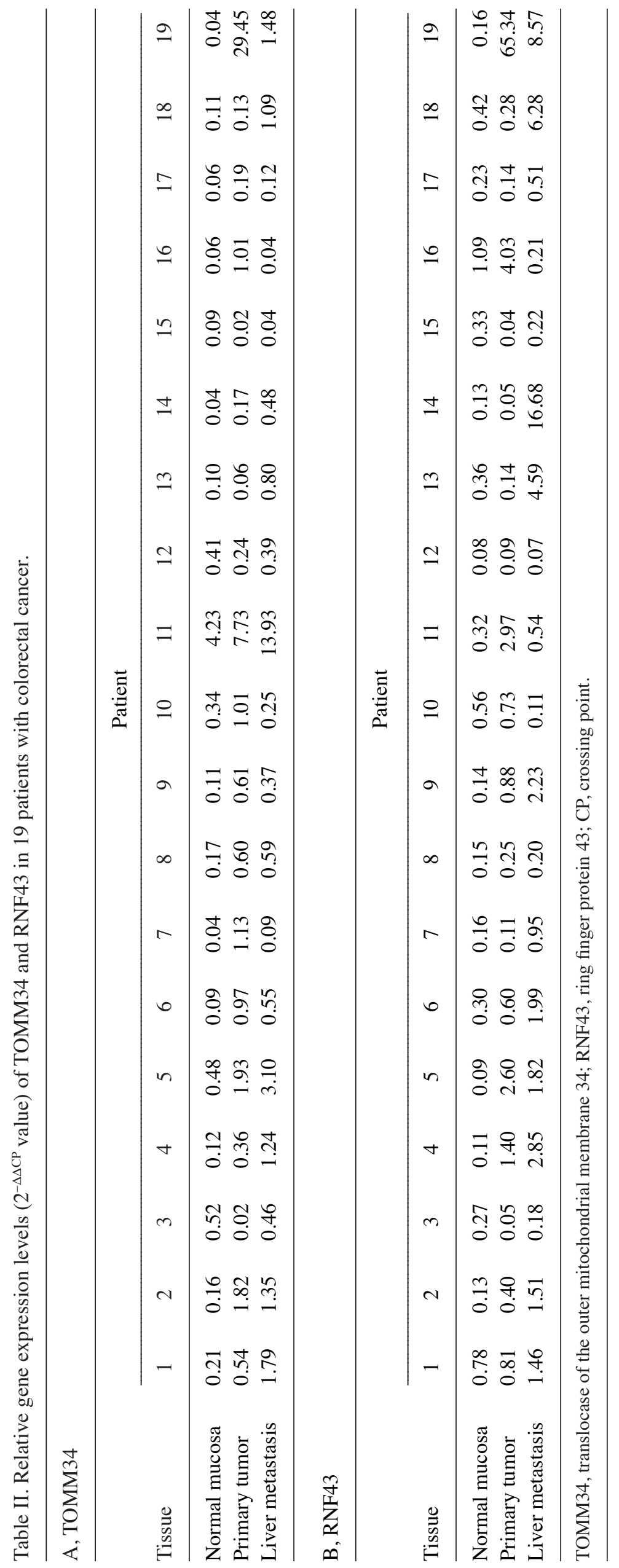


A

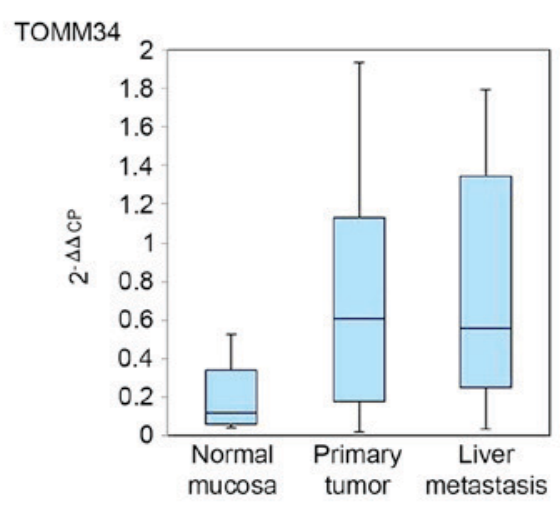

B

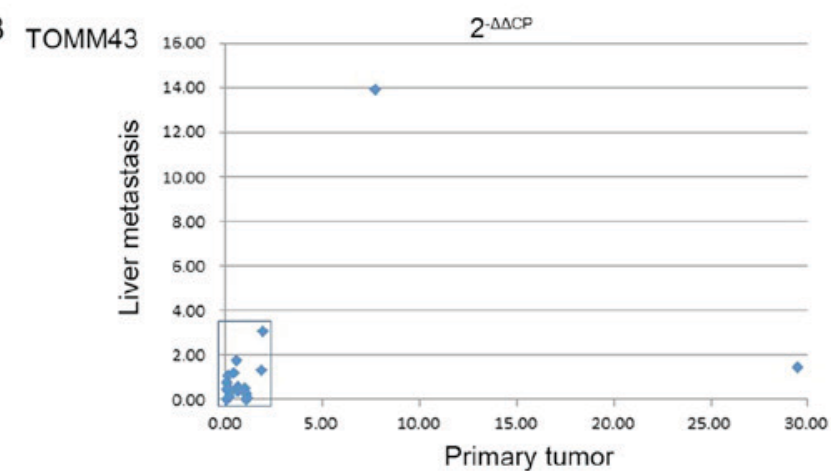

RNF43

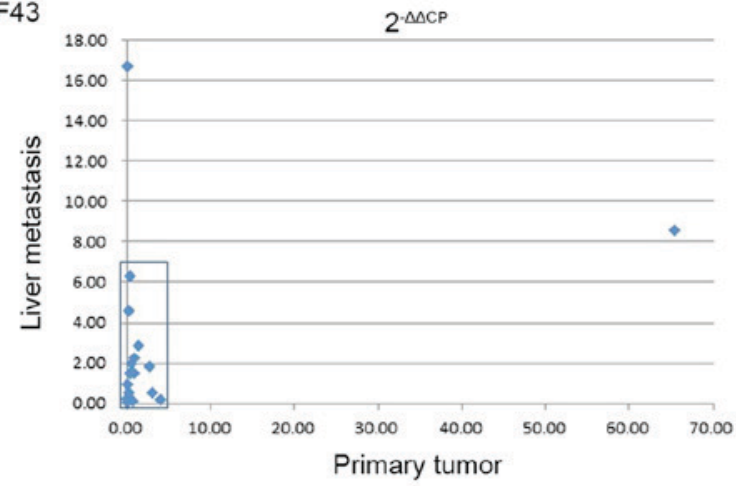

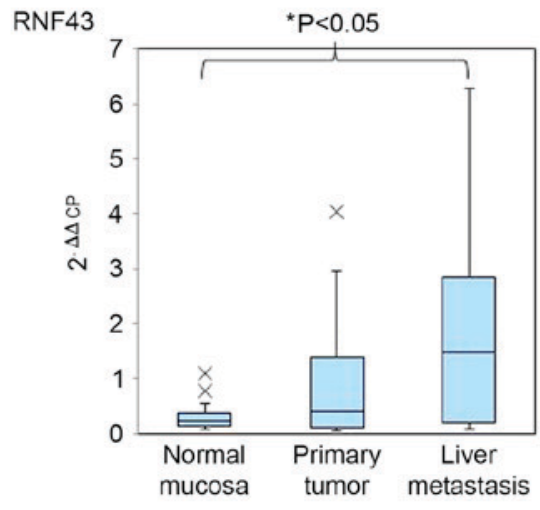

C томм34

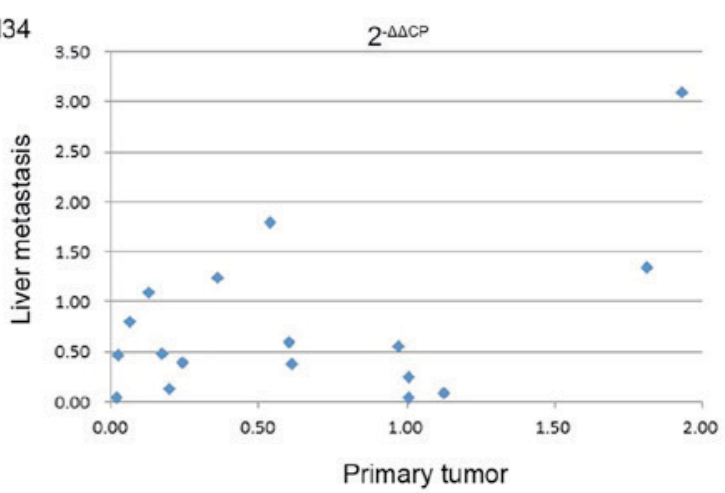

RNF43

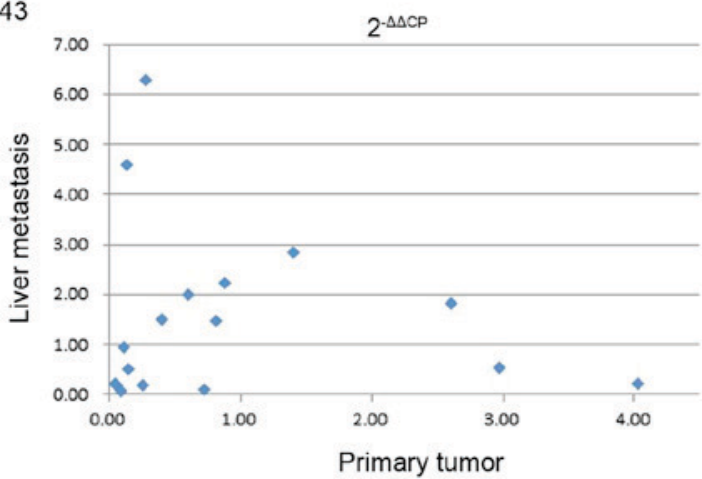

Figure 2. The relative gene expression level $\left(2^{-\Delta \Delta C P}\right)$ of TOMM34 and RNF43 in normal mucosa, colorectal primary tumor and liver metastasis was detected in samples from paraffin blocks by quantitative polymerase chain reaction. (A) Gene expression levels of TOMM34 and RNF43 in normal mucosa, primary tumor and liver metastasis samples. (B and C) Correlation of the gene expression levels between the primary tumor and liver metastasis. The gene expression level of all patients' primary tumors and liver metastases were plotted in (B). (C) Showed enlarged figure of squared area of (B). "P<0.05. TOMM34, translocase of the outer mitochondrial membrane 34; RNF43, ring finger protein 43; CP, crossing point.

area of Fig. 2B. There was no correlation of measured gene expression levels between primary tumors and liver metastases in both TOMM34 and RNF43 data.

\section{Discussion}

TOMM34 and RNF43 have been identified as oncogenes by comprehensive gene studies such as microarrays, and investigations of these genes may lead to the development of novel and effective molecular target drugs $(1,2)$. TOMM34 protein is present inside the nucleus and cytoplasm of tumor cells, whereas no expression is observed in normal organs, with the exception of the testes and ovaries $(10,11)$. High expression of TOMM34 is observed in colorectal cancer, which is considered to be associated with tumor growth, since cell proliferation is inhibited when the function of this gene is blocked by small interfering RNA (1). TOMM34 is also present in tumors such as hepatocellular carcinoma, lung cancer, bladder cancer, acute myeloid leukemia and soft tissue sarcoma (1). RNF43 is present at the endoplasmic reticulum and nucleus membrane; it is associated with cell proliferation and has ubiquitin ligase activity. High expression of RNF43 is observed in colorectal cancer and colorectal adenoma (2,12-14). In addition, RNF43 mutation has been reported in hepatocellular carcinoma, lung cancer, pancreas cystic tumor and cholangiocarcinoma (15-18).

Dendritic cells are antigen-presenting cells, and it is considered that these cells present cancer antigens to lymphocytes and induce tumor antigen-specific lymphocytes in vivo (19). 
A clinical trial of cancer vaccine therapy with dendritic cells pulsed with tumor lysate (used as an adjuvant postoperative treatment for patients with cholangiocarcinoma) demonstrated improvement of overall survival and relapse-free survival (20). Therefore, further development of such cancer vaccine therapies is expected.

Clinical trials of vaccine therapy using artificially synthesized cancer peptides based on the amino acid sequence of the tumor antigens TOMM34 and RNF43 are ongoing (3-6). A tumor antigen inoculated by vaccine is used for antigen presentation from dendritic cells to lymphocytes, and cytotoxic T-lymphocytes (CTLs) are induced in vivo. CTLs that recognize the tumor antigen show an anti-tumor effect against cancer cells expressing the tumor antigen. In fact, higher CTL induction was confirmed in proportion to the peptide concentration, and patients with a strong skin reaction at the vaccination site or a greater number of CTL responses to the peptide exhibited significantly longer overall survival $(4,6)$.

In light of these results, cancer peptide vaccine therapy appears to be a promising strategy by which treatment can be customized to the tumor antigen expression levels on each tumor. RT-qPCR can be conducted to quantify the gene expression of the tumor, and even a small amount of mRNA can be evaluated by this method. Using this assay, the present study was able to quantify the tumor antigen expression of an individual cancer, and a cancer peptide vaccine therapy could be tailored to the results. Furthermore, the correlation between the effectiveness of the vaccine and the gene expression level can be evaluated by measuring the gene expression in a stored specimen. However, mRNA from an old sample or a sample upon formalin fixation and paraffin embedding may be degraded by these procedures, and the quality of the mRNA may not be suitable for RT-qPCR.

In fact, TOMM34 and RNF43 expression could not be detected in samples derived from a paraffin block by qualitative PCR in our preliminary studies (data not shown). To the best of our knowledge, no study has attempted a quantification of TOMM34 and RNF43 expression in paraffin block samples. The present study was able to quantify the expression levels of TOMM34 and RNF43 mRNA upon extracting total RNA from primary colorectal cancer, liver metastasis and normal large intestine mucous membrane using the Universal ProbeLibrary assay. In this assay, the pair of probes created from 8-9 bases and the primers exhibited the specificity of the transcription product. Since the length of the PCR product was $<150 \mathrm{bp}$, it may be possible to perform this type of assay without the influence of RNA destruction.

The present study observed that the mRNA expression of TOMM34 and RNF43 in the primary tumor and liver metastasis tissue samples tended to be higher than that in the normal mucosa, but a significant difference was observed only for RNF43 between the liver metastases and normal mucosa, possibly due to an insufficient number of subjects. However, these oncogenes were confirmed to be present in the canceration process of the large-intestine mucous membrane, and effectiveness of a cancer vaccine therapy against a cancer that expresses those tumor antigens is expected. The gene quantitative methodology used in the present study may be useful for the refinement of cancer vaccine therapies based on tumor antigen expression. In future studies, the correlation between tumor antigen levels in stored samples and therapeutic outcomes will be evaluated.

In conclusion, although qualitative RT-PCR to confirm the expression of TOMM34 and RNF43 in paraffin-embedded samples was difficult to perform, the present study was able to quantify the expression levels of these genes using a Universal ProbeLibrary assay. This methodology may be useful for both customized cancer vaccine therapy based on tumor antigen expression levels and for the development of molecular targeting drugs. The present results confirmed that TOMM34 and RNF43 tended to increase in the canceration process of colorectal cancer in several-year-old samples stored in paraffin blocks. However, there was no significant difference in gene expression between the primary tumor and liver metastasis tissues in the canceration process. The present findings should be assessed in further studies with additional cases.

\section{References}

1. Shimokawa T, Matsushima S, Tsunoda T, Tahara H, Nakamura Y and Furukawa Y: Identification of TOMM34, which shows elevated expression in the majority of human colon cancers, as a novel drug target. Int J Oncol 29: 381-386, 2006.

2. Uchida N, Tsunoda T, Wada S, Furukawa Y, Nakamura Y and Tahara H: Ring finger protein 43 as a new target for cancer immunotherapy. Clin Cancer Res 10: 8577-8586, 2004.

3. Matsushita N, Aruga A, Inoue Y, Kotera Y, Takeda K and Yamamoto M: Phase I clinical trial of a peptide vaccine combined with tegafur-uracil plus leucovorin for treatment of advanced or recurrent colorectal cancer. Oncol Rep 29: 951-959, 2013.

4. Hazama S, Nakamura Y, Takenouchi H, Suzuki N, Tsunedomi R, Inoue Y, Tokuhisa Y, Iizuka N, Yoshino S, Takeda K, et al: A phase I study of combination vaccine treatment of five therapeutic epitope-peptides for metastatic colorectal cancer; safety, immunological response, and clinical outcome. J Transl Med 12: 63, 2014.

5. Yasuda S, Tsuchiya I, Okada K, Tanaka A, Suzuki T, Sadahiro S, Takeda K, Yamamoto S and Nakui M: Significant clinical response of advanced colon cancer to peptide vaccine therapy: A case report. Tokai J Exp Clin Med 37: 57-61, 2012.

6. Okuno K, Sugiura F, Hida JI, Tokoro T, Ishimaru E, Sukegawa Y and Ueda K: Phase I clinical trial of a novel peptide vaccine in combination with UFT/LV for metastatic colorectal cancer. Exp Ther Med 2: 73-79, 2011.

7. Srinivasan M, Sedmak D and Jewell S: Effect of fixatives and tissue processing on the content and integrity of nucleic acids. Am J Pathol 161: 1961-1971, 2002.

8. Miyazono Y, Kamogawa Y, Ryo K, Furukawa T, Mitsuhashi M, Yamauchi K, Kameoka T and Hayashi N: Effect of B7.1-transfected human colon cancer cells on the induction of autologous tumour-specific cytotoxic T cells. J Gastroenterol Hepatol 14: 997-1003, 1999.

9. Livak KJ and Schmittgen TD: Analysis of relative gene expression data using real-time quantitative PCR and the 2(-Delta Delta C(T)) method. Methods 25: 402-408, 2001.

10. Terada KJ, Ueno S, Yomogida K, Imai T, Kiyonari H, Takeda N, Yano M, Abe S, Aizawa S and Mori M: Expression of Tom34 splicing isoforms in mouse testis and knockout of Tom34 in mice. J Biochem 133: 625-631, 2003.

11. Chewawiwat N, Yano M, Terada K, Hoogenraad NJ and Mori M: Characterization of the novel mitochondrial protein import component, Tom34, in mammalian cells. J Biochem 125: 721-727, 1999.

12. Sugiura T, Yamaguchi A and Miyamoto K: A cancer-associated RING finger protein, RNF43, is a ubiquitin ligase that interacts with a nuclear protein, HAP95. Exp Cell Res 314: 1519-1528, 2008.

13. Takahashi N, Yamaguchi K, Ikenoue T, Fujii T and Furukawa Y: Identification of two Wnt-responsive elements in the intron of RING finger protein 43 (RNF43) gene. PLoS One 9: e86582, 2014.

14. Shinada K, Tsukiyama T, Sho T, Okumura F, Asaka M and Hatakeyama S: RNF43 interacts with NEDL1 and regulates p53-mediated transcription. Biochem Biophys Res Commun 404: 143-147, 2011. 
15. Yagyu R, Furukawa Y, Lin YM, Shimokawa T, Yamamura T and Nakamura Y: A novel oncoprotein RNF43 functions in an autocrine manner in colorectal cancer. Int J Oncol 25: 1343-1348, 2004.

16. Koo BK, Spit M, Jordens I, Low TY, Stange DE, van de Wetering M, van Es JH, Mohammed S, Heck AJ, Maurice MM and Clevers H: Tumour suppressor RNF43 is a stem-cell E3 ligase that induces endocytosis of Wnt receptors. Nature 488: 665-669, 2012.

17. Wu J, Jiao Y, Dal Molin M, Maitra A, de Wilde RF, Wood LD, Eshleman JR, Goggins MG, Wolfgang CL, Canto MI, et al: Whole-exome sequencing of neoplastic cysts of the pancreas reveals recurrent mutations in components of ubiquitin-dependent pathways. Proc Natl Acad Sci USA 108: 21188-21193, 2011.
18. Ong CK, Subimerb C, Pairojkul C, Wongkham S, Cutcutache I, Yu W, McPherson JR, Allen GE, Ng CC, Wong BH, et al: Exome sequencing of liver fluke-associated cholangiocarcinoma. Nat Genet 44: 690-693, 2012.

19. Sadanaga N, Nagashima H, Mashino K, Tahara K, Yamaguchi H, Ohta M, Fujie T, Tanaka F, Inoue H, Takesako K, et al: Dendritic cell vaccination with MAGE peptide is a novel therapeutic approach for gastrointestinal carcinomas. Clin Cancer Res 7: 2277-2284, 2001.

20. Shimizu K, Kotera Y, Aruga A, Takeshita N, Takasaki K and Yamamoto M: Clinical utilization of postoperative dendritic cell vaccine plus activated T-cell transfer in patients with intrahepatic cholangiocarcinoma. J Hepatobiliary Pancreat Sci 19: 171-178, 2012. 